\title{
Bernardus Marango.
}

\author{
Von \\ Hans v. Kap-herr.
}

Scheffer-Boichorst 1) hat die Annales Pisani auf die Autorität von Roncioni und Tronci dem Bernardus Marango zugeschrieben: Roncioni und Tronci, meint er, müssten handgreifliche durch einen Codex selbst gegebene Gründe gehabt haben, um Marango als Autor zu bezeicbnen. Langer ${ }^{2}$ ) geht einen Schritt weiter: er glaubt nachweisen zu können, dass sowohl der Verfasser der Anuales rerum Pisanarum ${ }^{3}$ ) als der Verfasser der Chroniche di Pisa 4) und Roncioni dieselbe Handschrift der Annales Pisani benutzt habe, welche uns noch jetzt vorliegt. Es wird sich ergeben, dass weder Roncioni noch Tronci unter dem von ibnen citierten Bernardus Marango die Annales Pisani verstanden haben.

Zum Jahre 1127 erzählt Roncioni (p. 233-234), dass die Genuesen das pisanische Castell Piombino erobert hätten, nur Bernardus Marango und Lorenzo Tajuoli sollen davon Nachricht geben: in den A. P. findet sich daron kein Wort. Dass der Papst den Pisanern im Jahre 1066 die Insel Corsica übergeben habe, und dass diese Vergünstigung den Zorn der eifersüchtigen Genuesen erregt habe, soll nach Roncioni (p. 127) nur bei Bernardus Marango zu lesen sein: die A. P. schweigen darüber.

Ebensowenig citiert Tronci die A. P. als das Werk des Bernardus Marango. Tronci (p. 92, 93, 107) führt die Consuln der Jahre 1159, 1160 und 1162 aus Bernardus Marango an: in den A. P. suchen wir sie vergeblich. Er erzählt (p. 140) zum Jahre 1174 über einen Vertrag zwischen Pisa und Venedig, Marango ist seine Quelle: in den A. P. findet sich nichts daron.

1) Forschungen 11, 513. 2) Politische Gesch. Genuas und Pisas im 12. Jahrhundert 209. 3) Ughelli Italia sacra ed. Coleti X Append. 99 ff. ๑) (Tartinius) Rerum Italic. SS. I, $311 \mathrm{ff}$. 
Ich könnte die Beispiele leicht vermehren. Wenn ich noch erwähne, dass von zwölf Fällen, da Tronci den Bernardus Marango anführt, sein Citat sechsmal auf solche Dinge geht, die nicht in den A. P. stehen, so ist der Beweis erbracht, dass Roncioni und Trouci unter dem Namen Bernardus Marango ein anderes Geschichtswerk vorgelegen hat als unsere A. P., und dass wir demnach kein Recht haben, sie nach Bernardus Marango zu benennen.

Wer ist aber der von den beiden Pisaner Geschichtsschreibern citierte Bernardus Marango? Coletti glaubte ihn im vorigen Jahrhundert gefunden zu baben. In dem ersten Bande der als Fortsetzung zu Muratori erschienenen Rerum Italicarum Scriptores gab er eine Chronik heraus, welche bis zum Anfang des 15. Jahrhunderts die Geschichte Pisas darstellt. Er glaubte nachweisen zu können, dass Tronci die "Chroniche di Pisa " als Bernardus Marango citiere, und er fand eine Bestätigung für üiese Autorschaft in der auffälligen Erwähnung, welche ein Bernardus Marango in ihnen findet. Zum Jahre 1159 (Chroniche p. 381) werden erst die Consuln und dann die Provisoren der Stadt Pisa aufgeführt, unter ihnen Bernardus Marango. "Dieser Marango," fährt der Autor der Chroniche fort, „war wohl zwölf Mal im Amte der Provisoren; wie alle diejenigen, welche zu diesem Amte ausersehen wurden, ein Mann von ungewöhnlicher Bedeutung und von grosser Vaterlsndsliebe ". Also, meint Coletti, war der Verfasser der Chroniche, der im 15. Jahrhundert lebte, hoch erfreut in ferner Vergangenheit einen Namensvetter gefunden zu baben, der eine so rühmliche Rolle in der Geschichte seiner Vaterstadt gespielt hat; er versäumte nicht in wohlberechtigten Stolze die Verdienste seines Ahnherrn ins Licht zu setzen. Dagegen hat dal Borgo ") eingewendet, dass die Citate Troncis doch nicht so genau auf die Chroniche passen, und dass Tronci selbst den Bernardus Marango als eine lateinisch geschriebene Chronik bezeichnet. Wir können hinzufügen, dass nach Roncionis Aussage die Annalen des Bernardus Marango nur bis zum Jahre 1175 reichten.

Aber vielleicht ist die auffällige Hervorhebung des Bernardus Marango nicht auf Rechnung des Autors der Chroniche, sondern auf Rechnung seiner Quelle zu setzen. Sollte diese Quelle identisch sein mit dem bei Roncioni und Tronci citierten Bernardus Marango? Thittsächlich finden wir die meisteu der von Roncioni und Tronci dem Beruardus Marango zugeschriebenen Notizen in den Chroniche wieder. Dazu ist die von Coletti bemerkte Hervorhebung des Beruardus Marango

t) Dissertazioni sopra l' historia Pisana I 1, 118 ff. 
keineswegs vereinzelt. Die Chroniche pflegen zu Anfang eines Jahres die Cousuln der Stadt anzuführen; selten werden auch andere Beamte, Provisoren und Richter erwähnt, nur fünf Fälle sind mir (in der Zeit bis 1175) bekannt: in zweien fungiert Bernardus M. als Provisor (p. 381-400); in einem dritten erscheint Salome figliulo di Bernardo Marangoni unter den Richtern (p. 390); bei dem vierten wird wieder der Sohn Bernardos genannt, ob unter den Provisoren oder den Richtern, lässt sich nicht erkennen 1); in dem fünften erscheint ein Salome unter den Richtern, der doch wohl mit dem Salome Marangone identisch ist.

So liegt also die Vermuthung nahe, dass sowohl in den Chroniche als bei Roncioni und Tronci eine verloreve lateinische Chronik benutzt ist, die eben jenen Bervardus Maraugo, der in der pisanischen Geschichte des 12. Jahrhunderts eine nicht unbedeutende Rolle spielt, zum Verfasser hat. Und in der That bemerken wir in den Chroniche und übereinstimmend bei Roncioni und bei Tronci Nachrichten, welche sich in den erhaltenen Pisaner Chroniken nicht finden, Nachrichten deren Richtigkeit wir urkundlich bestätigen können, und die doch weder aus Urkunden noch aus nicht-pisanischen Quellen geschöpft zu sein scheinen.

Am auffälligsten ist die Anführung der Consuln zu Beginn des Jahres. Dass die Consullisten aus Bernardus Marango stammen, wird uns für drei Jahre von Tronci (p. 92, 93, 107) bezeugt, für andere Jahre ist es aus der oben besprochenen Bevorzugung des Bernardus Marango in den Beamtenlisten der Chroviche zu schliessen. Die Aunahme ist nicht zu kühn, dass Bernardus Marango ein vollstäuldiges Verzeichniss der pisanischeu Consuln enthalten hat.

Ich wende mich zu Nachrichten anderer Art. Die pisanischbyzautinischen Verträge in der Zeit ron 1111-1170 sind uns nur aus dem Vertrag zwischen Pisa und 'dem Kaiser Isaac Angelus vom Jahre $1192^{2}$ ) bekannt, in welcheu die früheren Verträge theilweise aufgenommen wurdeu, und zwar ein Vertrag zwischen Pisa und Alexius vom Jahre 1111 (p. 52-54), und eine Bestätigung desselben durch Kaiser Manuel von 1170 (p. 54). Zwischen diesen beiden Verträgen liegt ein Vertrag Pisas mit Kaiser Manuel, der in der Urkunde nur

1) Die Stelle ist verstümmelt; von den Namen der Consuln ist nur der erste erhalten; er beweist, dass die Consuln d. J. 1164 gemeint sind, welche wir aus einer Urkunde, (bei Bonaini Stat. ined. 41) kennen. Salome Marango ist nicht unter ihnen: also kann er nur unter den Judices oder Provisores gewesen sein, die in der Chroniche nach den Consuln autgeführt werden. $\because$ Documenti sulle rolizianoni delle città toscane etc. ed G. Müller $40 \mathrm{ff}$. 
ganz beiläufig erwähnt wird: Pisa verpflichtet sich im Jahre 1170 alles zu erfüllen, was es in den Verträgen mit Alexius und mit Mannel versprochen habe ${ }^{1}$ ). Ueber diesen früheren Vertrag mit Manuel berichtet nun Roncioni (p. 256), dass der pisanische Senat nach dem Todes des Kaisers Johannes Ugo Dodone, der als Vorstand der pisanischen Colonie in Byzanz weilte, brieflich beauftragt habe dem neuen Kaiser Manuel die Theilnahme der pisanischen Regierung an dem Verlust seines Vaters auszudrücken. Damals sei der alte Vertrag erueuert worden. Von diesem Ugo Dodone weiss Roncioni weiterbin zu berichten, dass er im Jahre 1137 (c. P.) mit den byzantinischen Gesandten, welche Kaiser Johannes damals nach Pisa geschickt hatte, nach Byzanz gereist sei, und dass er dort das Amt des pisauischen Consul bekleidet habe. Nur von der hyzantinischen Gesandtschaft erzählen die Annales Pisani ${ }^{2}$ ), nichts von der Sendung des Ugo Dodoue, aber der Bericht Roncionis findet eine Bestätigung durch eine 1141 in Byzanz ausgestellte Orkunde, in welcher Ogo Dudonis, qui tunc erat legatus Pisanus ${ }^{3}$ ), als Zeuge fungiert.

Ebenso wie über die byzantinisch-pisanischen Verhandlungeu werden wir über die Beziehungen zwischen Venedig und Pisa nur durch einen späteren Vertrag unterrichtet. In einem Documente vom Jahre $1180^{4}$ ) bezieht sich der venetianische Doge auf einen Vertrag, den sein Vorgänger Sebastianus Ziani mit dem pisanischen Gesandten Bulgarinus Anfossi abgeschlossen hat. Zum Jahre 1174 erzählt uns Tronci, wie er angiebt aus Bernardus Marango, dass Bulgarinus Anfossi nach Venedig geschickt worden sei, um einen Vertrag abzuschliessen,

1) Die Existenz dieses Vertrages ist weder von Heyd noch von Langer be. achtet worden.

2) Für welche Roncioni das Datum 12. Angust angibt, das in den A.P. fehlt; er kann es aus den Chronichette Roncioni entnommen haben (Codex Sardiniae 1.659), die er sonst auch benutzt. Es wire in unserer Bandechrift wohl nur durch einen Schreibfehler aurgefallen sein, denn die Chronichette benützen die Annales Pisani an dieser Stelle. Dagegen möchte ich die Angabe Roncionis, dass die 200 Pallien zum Ersatz für die von Kaiser Alexius im Jahre 1111 versprochenen Pallien und Gelder gezablt wurden, für unsere Quelle beanspruchen. Jedenfalls hat sie Roncioni nicht aus dem Vertrage von 1170 erschlossen, den er gänzlich missverstanden hat (vgl. Roncioni 873). Es ist übrigens gar kein Grund die Zahl der 200 Pallien zu beanstanden, wie das Langer 203 thut. Sind die Ebrengeschenke seit 1111 nicht gezahlt worden, so war der Kaiser is Pallien und 2500 Byz. schuldig. Vermuthlich trat die höhere Zahl von Pallien an die Stelle der geschuldeten Gelder. Keinesfalls ist es möglich die Zahl 200 auf einen Febler der Copisten der A. P. zurückzufïhren, denn auch die von Chronichette Roncioni benutzte Handschrift giebt die Zahl 200.

9) Documenti etc. 4. अ Documenti etc. 21-22. 
dessen Bediugungen er näher angiebt. Am 8. September sei Anfossi mit Giovanni Duodi dem venetianischen Gesandten aus Venedig zurückgekehrt. Ebenso wird diese Verbandlung bei Roncioni erzählt (p. 391 bis 392). Fünf Jahre früher, so berichten uns diesmal Chrouiche (p. 427), Roncioni (p. 363) und Tronci (p. 129), sei zwischen Pisa und Venedig ein Vertrag auf fünf Jabre abgeschlossen worden. Die pisauischen Consuln Ildebrando Bamboni (di Parlascio bei Tronci) und Ugoni Orlandi seien als Gesandte nach Venedig geschickt worden; der Vertrag sei im Mai abgeschlossen worden. Roncioni berichtet dazu noch einige Details über die Ursacheu des Streites zwischen Pisu und Venedig ${ }^{1}$ ).

Dass Roncioni und Tronci in diesen Fällen Orkunden verwerthet hätten, ist wenigstens in dem einen Fall, da Tronci den Bernardus Marango citiert, ausgeschlossen. Unwahrscheinlich ist es auch, dass sie die Namen der Gesandten, der pisauischen sowohl als der venetianischen, aus dem Text der Verträge erfahren bätten. Ich werde jetzt einen Fall anführen, bei dem mir die Benutzung von Orkuudeu gänzlich unmöglich scheint.

Aus den A. P. wissen wir, dass im Jahre 1170 eine pisauiscle Gesandschaft nach Sicilien gegangen ist. Die Chroniche (p. 428) erzählen (ähnlich Roncioni p. 364), dass sich ihr auf der Rückkehr sicilianische Gesandte, der Grancancelliere Rinaldus ${ }^{2}$ ) und Rubertus, angeschlossen haben; bei Gaeta treffen sie auf ein genuesisches dem Bisaccia gehöriges Schiff, welches reichbefrachtet aus Byzanz heimkehrt. Die Pisaner kapern es, liefern es aber den sicilischen Gesandten aus, welche sich darauf berufen, dass die Galeere auf sicilischem Gebiete genommen sei. Es geschah dies im November. An 17. Dezember traf die pisanisch-sicilianische Gerandschaft in Pisa ein, am 15. Januar wurde daselbst der Friede beschworen. Alles dies wissen wir nur aus den Chroniche und Roncioni.

Der Name des Bisaccia ist uns aus der genuesischen Geschichte kaum bekannt: im Jahre 1181 und 1192 erscheint ein Bisacia unter den genuesischen Consuln ${ }^{3}$ ); wohl aber wissen wir aus genuesischen

1) Roncioni sagt, die Venetianer seien auf die Pisaner eifersüchtig gewesen, weil ihnen der Kaiser bessere Plätze im Hippodrom angewiesen habe. Welcher Werth auf die Theilname an den Festspielen gelegt wurde zeigt der Umstand, dass sich die Pisaner im Vertrage mit Kaiser Alexius im Jahre 1111 ausdrück. lich Plätze im Hippodrom ausbedungen (vgl. Documenti 53). 2) Wenn um diese Zeit kein sicilischer Kanzler Rinaldus nachweisbar ist, so man darf daraus bei der schlechten Textesüberlieferung der Chroniche, die namentlich die Namen stark entstellt hat, keine Schlüsse zielen. ${ }^{3}$ ) Ottoboni Annales 99 und 106. Ein Bisatia erwähnt Oberti Ann. 86 . 
Privaturkunden, dass ein Bisaccia nach Constantinopel Handel trieb, im Jahre 1160 schloss er in Genua mehrere Verträge ab, die sich auf seine Handelsreise nach Byzauz beziehen. (Chartae II, 624, $651,657$.

Freilich sind nicht alle Nachrichten, welche die Chroniche und Roucioni über die Annales Pisani hinaus bringen, von der gleichen Beschaffenheit. Auch bei solchen Angaben, welche Roncioni und Trouci nach eigener Aussage aus Bernardus Marango entnehmeu, lässt sich eine späte Entstehung unzweifelhaft nachweisen. Nach Bernardus Marango war Pelops der Gründer Pisas (Roncioni p. 10), der heil. Petrus der Gründer der pisanischen Kirche (Roncioni p. 30). Die Nachricht, dass Pelops Pisa gegründet, findet sich zuerst bei dem Predigermönch Bartholomaeus de Sancto Concordio, welcher in 14. Jabrhundert ein Buch de origine civitates Pisanae schrieb 1). Dass der heil. Petrus in Pisa gewesen sei, lesen wir zuerst in den Anuales rerum Pisanarum, die nach 1267 verfasst sind. Bei Marango soll auch nach Roncioni (p. 255) gestanden baben, dass die Pisaner im Jahre 1140 Neapel erobert und sieben Jahre im Besitz gehabt, eiue sagenhafte Nachricht, die sich zuerst im Chronicon breve (Ughelli X, 118) findet, und ans diesem in das Breviarium des Michael de Vico übergegnngen ist. Von gleicher Art ist die Eroberung von Lipari durch die Pisaner im Jahre 1035 (Ronsioni p. 81 aus Marango), und die Abtretung der Insel an den römischen Kaiser; von gleicher Art die Belehnung Pisas mit Corsica durch den heilige Stuhl im Jahre 1089.

Wem hieraus noch uicht die späte Entstehung ler Chronik des Beruardus Marango einleuchten sollte, den verweise ich darauf, dass Bernardus Marango wach Roucioni (p. 285 und 316) eine Umrechnung der pisanischen Münzen in Goldflorinen vollzogen hat: der erste Goldflorin wurde im Jahre 1252 geprägt ${ }^{2}$ ), Bernardus Marango kann sein Werk erst geschrieben baben, als das Florentiver Gold die Alleinherrschaft in Pisa errungen hatte.

1) Ughelli ג, 118. Dass die Nachricht aus Bartholumäus de Sancto Concordio entnommen ist, ergiebt sich aus der Anführung der Stelle im Breviarium des Nlichael da Vico (Muratori VI, 165), wo der Text vollständiger erbalten ist als bei Ughelli. 2) Vgl. Giovanni Villani Istoria forentina VI, ji ed Dragomanui. Der Goldflorin gewann schon in den ersten Jahrzehnten des 14. Jahrhunderts in Italien allgemeine Verbreitung. In Pisa wurde er damals nachgemacht. Vgl. Claudius Boissinius: De valore Fioreni aurei Fiorentini in Argelatus Dissertatione de monetis Italiae IV, iv4-106, wo die Cilate aus Vilani zubammengestellt sind. 
Recapitulieren wir: Bernardus Marango ist eiue lateinisch geschriebene Compilation 1), vicht vor dem 14. Jahrhundert verfasst; sie reicht bis zum Jahre 1175 und begünstigt in auffallender Weise deu Bernardus Marango, einen pisanischen Staatsmann des 12. Jahrhunderts. Sie benutzt die Aunales Pisani, daneben wahrscheinlich schon das Breviarium des Michael de Vico oder doch seine Quellen ${ }^{2}$ ), und verwerthet neben mancherlei Sagenhaftem aus anderen Quellen oder aus eigener Phantasie ein Verzeichniss der pisanischen Beamten und eive zeitgenössische Quelle ${ }^{3}$ ).

Offenbar eine höchst seltsame literarische Erscheinung! Dass sie sich für das Werk eines Bernardus Marango ausgegeben hat, scheint mir nach dem übereinstimmenden Zeugniss von Roncioni und Tronci nicht zu bezweifeln. Wie würde sich sonst anch die auffallende Hervorhebung des Bernardus Marango und seines Sohnes in den pisanischen Stadtämtern erklären lassen? Ob aber ein gleichnamiger Nachkomme des pisanischen Stidtbeamten die Chronik verfasst hat, oder ob ein Fälscher sein Machwerk für alte pisanische Annalen ausgegeben hat, diese Fragen zu entscheiden mangelt es mir an Material. Ich will aber bemerken, dass Dal Borgo (Dissertazione I, 122) den Namen Marango in den Registern des Archivs cle Sig. Priori, weluhe die adeligen und bürgerlichen Familien Pisas enthalten, nach dem Jahre 1300 vergeblich gesucht hat.

Ess würde nun meine Aufgabe sein, jener verlorenen Chronik nachzuforschen, die der verlorene Bernardus Marango benutzt hat. A ber auf ibre Lösung müsste bei dem mir zugänglichen Material von vornherein verzichtet werden. Leichter schon dürfte eine Prüfung der neuen Nachrichten in den Chroniche, bei Roncioni und Tronci sein, ohne Rücksicht darauf, dass sie gerade aus Bernardus Marango stamneu. Deun Roncioni benutzt ueben Bernardus Marango noch

1) Der Titel lautete Annales Pisani (Roncioni 100), wahrscheinlich sind es die in den Chroniche 864 citirten Annali Pisani; es wird mehrfach als eine kurze Quelle bezcichnet (z. B. Roncioni 1. c.). Allerdings ist darauf nicht viel zu geben, da Roncioni daneben sehr umfassende Compilationen vorgelegen haben müssen. 2) Ob das Breviarium selbst benützt ist, wird sich kaum feststellen lassen, da is nichts Originales enthält. Dass übrigens die Ann. Pisani und nicht etwa llloss das Breviarium benutzt sind, beweist 2. B. die Anfïhrung bei Tronci 8i; die hier erwäbnten baugeschichtlichen Notizen finden sich nur in den A.P., nicht in den Annales rerum Pisanarum, und nicht in Breviarium. 3) Dass das Beamtenverzeichniss unabhängig von der verlorenen Quolle gewesen sei, schliesse ich daraus, dass in den Chroniche und bei Roncioni die Nachrichten über die pisanische Beamtenschaft nicht wit dem Jahre 1175 abbrechen. 
die Origines des Bartholomaeus de Sancto Concordio ${ }^{1}$, das Geschichtswerk des Lorenzo Tajuoli ans Pistoja und die Cummentarii urbani des Raffaele da Volterra ${ }^{2}$ ); auch aus diesen mag wohl eine brauchbare Notiz in seine Compilation übergegangen sein - und ich möchte keineswegs behaupten, dass die glaubhaften Nachrichten, welche ich oben für deu vermeintlichen Bernardus Marango des 12. Jahrhunderts beansprucht habe, sämmtlich aus dem wirklichen Bernardus Marango entrominen sind, den wir jetzt als den Autor des 14. Jahrhunderts erkaunt habeu. Aber auch die Lösung dieser Aufgabe überlasse ich dem Herausgeber der Pisaner Annalistik in den Monumenten; ihm

1) Das Buch des Bartholomäus de Sancto Concordio wird in dem, aliud fragmentun " des Chronicon breve Pisanum (Ughelli $X, 118$ ) benutat. Vollstïndiger als in der Ausgabe Ughellis findet sich die Stelle in Breviarium. Hier stehn an Schlusse der betreffenden Notizen die Worte: , Explicit liber de origine civitatis Pisanae compositus a fratre Bartholomaeo de Sancto Concordio ordinis Predicatorum * (vgl. Scheffer-Boichhorst 527 n. 2). Bartholomäus de Sancto Concordio wird von Roncioni und Tronci (15) als Bartbolomäus Spina citirt. diesem wird das Buch , dell' origine di Pisa" zugeschrieben. Das von diesen benützte Werk handelte übrigens nicht blose über die Anfänge Pisas, sondern gab eine Geschichte bis tief im 13. Jahrhundert. Die Bezeichnung als Bartholomäus Spina beruht auf eine Verwechslung mit dem maestro del sacro palazzo Bartolomeo Spina, welcher cbenso wie Bartolomeo de Sancto Concordio ein pisaner Predigernönch war, aber im 16. Jahrhundert lebte (Roncioni 99: Bartolomeo Spina in libretto, che fa dell' origine di Pisa.... fiori nel mille cinquecento maestro del sacro paiazo di Roma). Dieser schrieb einen Tractatus de strigiis et lamiis, welcher von Runcioni $(\mathbf{c} 0)$ und Tronci $(i)$ als ein, libro della questione delle streghe citirt wird. So erklärt sich leicht der Irrthum. - Man könnte einen Augenblick versucht sein in dem Breviarium die Origines des angeblichen Bartholomäus Spina zu crblicken, - denn, dass Michael da Vico mehr gewesen sei als der Abschreiber der Chronik, hat er durch seine Unterschrift eigentlich nicht gesagt. Die letzte Erwähnung Spinas findet sich bei Roncioni 552 bei einem Ereigniss des Jahres $126 \%$, aber sie gerade beweist, dass nicht das Breviarium gemeint sein kann. Es sull nïmlich bei Spina gestanden baben, dass Graf Giordano der damalige Generalvicar von Tuscien bei der Belagerung von Fucecchio zugegen gewesen sei. Diese (ūbrigens. falsche) Angabe findet sich nicht im Breviarium. - Ueber Bartholomäus de Sancto Concordio vgl. Quétif et Echard SS. Ordinis Predicatorum I, $293 \mathrm{ff}$. (Fineschi) Memorie istoriche Pibane III, 109-146. Cronaca del Convento di San Catarins mit den Anmerkungen von Bonaini im Archivio scorico VI, Q, $521 \mathrm{ff}$. Er ist 1262 geboren und 1847 gestorben. Mehrere Werke sind von ihm gedruckt, die Origines finde ich nirgends erwähnt. - Ueber Bartholomäus Spina vgl. SS'. Ordinis Predicatorum Il 1.6 u. ff. (Fineschi) Memorie III, 269 u. $28 \%$. Fineschi spricht 283 von einer handschriftlichen Chronika Pisana Spinas: wahrscheinlich kennt er sie nur aus Roncioni oder Trosici.

2) $\mathrm{i} 522$ in Rom, Seine Commentarii sind mehrfach gedruckt. Rom 1506, Paris 1526. 
wird neben mehrereu ungedruckten Pisauer Aunalen, wie sic schon ron Bethmann für die Monumente abgeschrieben sind 1), hoffentlich ein Exemplar der von Bonaini gedruckten, aber nie herausgegebenen Pisauer Urkunden zu Gebote stehn, die wir schmerzlich vermissen; er wird die Commentarii urbani des Raffaele de Volterra benutzen können, vielleicht gelingt es ihm auch die handschriftliche Chronik des Lorenzo Tajuoli, welche noch nach dem Jahre 1839 vou Valtancoli Montazio in Pisa benutzt wurde, zu Rathe zu ziehen ${ }^{2}$ ).

Doch seien mir einige Bemerkungen gestattet, die für denjenigen, welcher etwa eine vereinzelte Notiz aus den Chroniche und aus Roncioni verwerthen wollte, von Nutzen sein könuten. Ein bedeutender Gewinu für die pisanische Geschichte wird sich aus einer Erforschung der verlorenen Quelle des Bernardus Marango kaum ergeben: es ist nicht eine breite, zusammenhängende Darstellung, etwa eine weitere Fassung der Aunales Pisani, sondern es sind immer nur vereinzelte Nachrichten: wenn die Chroniche und Roncioni Nachrichten der A. P. ausführlicher wiedergeben, so ist ihr Detail so wenig charaktesistisch, so einförmiger Art, dass man überall den ausschmückenden Bearbeiter erkennt. - Die Chroniche sind übrigens eine der denkbar geistlosesten Compilationen; sie erzählen die meisten Thatsachen doppelt, zunächst aus dem Breviarium, dann noch einmal aus einer umfassenderen Quelle, wahrscheinlich eben unserem Bernardus Marango; die Thatsachen erscheinen unter falschen Jahren, und oft dermassen entstellt, dass man sie nur mit Mühe wiedererkennt.

Im Allgemeinen ist die Fassung bei Roncioni vorzuziehen, aber nicht immer, denn Roncioni scheint eine von den Chroniche noch nicht gekannte Bearbeitung, wahrscheinlich das Werk des Lorenzo Tajuoli benutzt zu baben, welches besonders in den älteren Partien mit den ruhmredigsten Lügeu geschmückt ist: mit Vorliebe werden Namen für pisanische Feldherrn aus dem 10 und 11. Jahrhundert erfunden ${ }^{3}$ ), lauge Reden werden ihnen in den Mund gelegt, und die Schlachten gegen Sarazenen und Genuesen sind mit livianischer Austührlichkeit und Eintönigkeit beschrieben. Der entstellende Einfluss dieses Werkes erstreckt sich, wenn auch weniger merklich, bis in

1) Archiv XIl, ร84, s85, 642, ๆ08, 732. 2) Vgl. Annali Pisani di P. Tronci da Valtancoli Montazio. Secunda ediz. da Giovanni Sforza Pisa 1868. I, 13̀, 100. Die in Lucca nach $18 \% 9$ erschienene Originalausgabe kenne ich nicht. 8) Man vergleiche z. B. die Sarazenenkämpfe bei Roncioni p. 41-47, p. 48 ff. und 54-57 mit dem entsprechenden Partien der Chroniche p. 316 ff. Der Feldherr Carlo Orlandi, der Consul (!) Philippo Visconti. 
das 12. Jahrhundert, gelegentlich werden Namen, die in den Chroviche aus den Annales Pisani entnommen sind, hier vollstäudig verändert ${ }^{1}$ ). Aber auch schon in den Chroniche scheinen viele Namen willkürlich beigefügt zu sein ${ }^{2}$ ); wir müssen uns hier überall durch ein dichtes Netz von Lüge und Irrtbum durciarbeiten ${ }^{3}$ ).

Bekannt ist, dass Roncioni eine grosse Anzahl von Urkunden benutzt. Auch der Verfasser der Chroniche kennt viele Orkunden, auch er hat wie Roncioni die Absicht, seinem Werke einen Urkundenanhang zu geben (p. 3\%0, vgl. auch z. B. 379, 398). Roncioni oder eine seiner Quellen hat eine gewisse Fertigkeit in der Benutzung vou Urkunden: er hat z. B. p. 367 richtig herausgefunden, dass der Bau des Castello auf der Via regia gegen eine Bestimmung des den Pisanerı von Friedrich ertheilten Privilegs verstiess. Darum ist Vorsicht geboten, wenn der Wortlaut bei Roncioni die Benutzung einer Orkunde wahrscheinlich macht, denn eine solche Fertigkeit kann ebensowohl der Wahrheit dienen, als in der Hand eines Fälschers Unheil stifteu.

So würde ich z. B. p. 301 aus dem Passus im Treuschwur des Tolomeus , che tutte le mercanzie, che per naufragio capitauero velle sue marine, e salva; si condussero al lido, egli liberamente gliele restituerebbe" nicht olne weiteres ammehmen, dass Roncioni (oder seine Quelle) den Treuschwur wirklich gekaunt habe, denn die angeführte Bestimmung findet sich sehr häufig in Handelsverträgen der Zeit. Auch darf der Vertrag mit dem „König von Septa “, dev Roncione p. 350 anführt, nicht ganz gläubig acceptirt werden; er enthält nur die bei den Verträgen Pisas mit fremden Staaten allgemein

1) Z. B. werden nach den A. P. p. 254 und den Chroniche p. 409 im Jahro: 1167 die Consuln Feriolo und Vettulario nach Sardinien geschickt, bei Roncioni erscheinen für dieselbe Mission die Consuln Stefano Mosca und Pietro Erici. Diesu Namen sind aus dem Cousularverzeichniss entnommen, wie es Ronciuni anfïhrt: die Namen Feriolo und Vettulario fehlen darin, in dem vollständigeren Verzeichniss dagegen, wie es die Cbroniche aus Bernardus Marango (dass es an B. M. stammt, beweist die Erwähnung des Salome unter den Provisoren) anführten. sind sic cuthalten.

2) So lernen wir in der Chroniche p. 401 und bei Roucioni p. 540 einen jisunischen Admiral Gherardo Gusmari, und einen Feldherm Hugo Pagani keunen, von denen wir sonst nichts wissen. Roncioni p. iso und Chroniche p. $4 \$ 0$ wird zu Marzucus liaetani und Albertus Bulsi, die wir aus den A. P. p. 녕 als Feld. herrn ler Pisaner kenuen. hiuzngefügt: Alamannus Duodi und Ugone Bella.

3) Ich will damit keineswegs ausschliessen, dass sich nicht auch für dic Nawen hie und da aus Roncioni eine Textverbesserung zu den A. P. ergeben sollte: so ist z. B. p. 265 statt cum duobus consulibus Pane et Porro videlicet et Carone zu lesen Paneporro videlicet et C. vgl. Roncioni 889 . p. 241 statt Miaifitram zu lesen Malf, Trani bätte es der Hülfe Roncionis uicht bedurlt. 
üblichen Bedingungen. Es scheint mir auch zweifelhaft, ob Roncioni die p. 363 angeführte Verpflichtung des Grafen Ildebrandino wirklich in dem Friedensvertrage gefunden hat.

Ich kehre zurück zu den Annales Pisani. Mit der Autorschaft des Bernardus Marango wird auch das Zeugniss Roncionis hinfällig. dass die A. P. mit dem Jahre 1175 ibren Abschluss gefunden haben. Aber wir kennen jetzt zwei selbständige Ableitungen aus den A. P. ${ }^{1}$, welche bis zum Jahre 1175 reichen, und so würde sich hieraus die Vermuthung ergeben, dass sie doch mit dem Jahre 1175 geendet haben. Damit ist noch nicht gesagt, dass sie ron einem Zeitgenossen herrühren; ich werde einige Momente zusammenstellen, welche gegen diese Annahme zu sprechen scheinen.

Im Allgemeinen schreiten die A. P. streng chronologisch vor, nur an zwei Stellen machen sie eine Ausnahme: beim Jahre 1154 reichen sie mit einigen Notizen bis zum Jahre 1162, und beim Jahre 1157 greifen sie bis zun Jahre 1167 vor: beide Mal sind die von ihnen gebrachten Nachrichten durchweg localgeschichtlichen Inhalts, sie handeln über den Bau von Kirchen und Stadtmauern, über Feuersbrünste, Hagelschlag und Theuerung: es liegt die Vermuthung nahe, dass der Verfasser hier eine Pisaner Stadtchronik von beschränktem Gesichtskreise benutzt hat. Dies würde noch keineswegs gegen eine Abfassung etwa in den 80 er Jahren des 12. Jahrhunderts sprechen (das Jahr 1180 ist das letzte in den Annales Pisani angeführte Datum). Auffallend ist es aber, dass die hier zusammengestellten Notizen im spätern Verlaufe der Darstellung bei den zugehörigen Jahren theilweise wiederkehren. P. 242 wird uns erzählt, dass der Baumeister Conectus im Jahre 1159 drei Säulen zum Dombau aus Elba herbeigeschafft habe. Dasselbe kann man in anderem Zusammenhange p. 244 lesen. Dass der Consul Coccus im Jahre 1159 zwei Thürme im Hafen Magnalis begonnen und die Quelle von St. Stefano zum Nutzen der Seeleute ummauert habe, steht auf p. 243 und nochmals auf p. 244. Dass

1) Dass Bernardus Marango nicht etwa dieselbe Handschrift der A. P. beniitzt hat, die uns vorliegt, beweisen die richtigeren Lesungen, die er bietet. Vgl. $\%$ den obeu angeführten Beispielen noch A. P. p. 24i. In der Handschrift steht: in mense Madii Pisani galeas quadraginta facere inceperunt et per totum inensem fuere Martinm complete. Pertz hat corrigirt Martii statt Madii. Langer p. 8! n. 2 corrigirt Madium statt Martium. Aber es wäre doch kaum denkhar, dass die Pisaner wirklich 40 Galeeren in einem Monat gebaut hätten. Roncioni p. 813 erzählt, dass sie die Galeeren in 11 Monaten fertiggestellt hätten. Es ist aber etwa zu lesen: et per totum [annum laboravernnt: usque ad] mensem Martium complete fuere. 
diese Thürme in den Jahren 1163 and 1165 vollendet wurden, wird uns p. 243 und p. 247 erzählt.

Sollten einem Zeitgenossen diese für dev Pisaner Bürger wichtigen Tagesereignisse so geringen Eindruck gemacht haben, dass er es nicht bemerkte, wenn er sie zweimal erzäblte?

Aber nicht bloss auf localgeschichtlichem Gebiete finden sich Wiederholungen. Zum Jahre 1159 erzählen die Anualen dasselbe Ereigniss zweimal hintereinander. Ich stelle die Worte nebeneinander, so wie sie nacheinander folgen:

Eodem anno $15 \mathrm{Kal}$. Septe mbris consules miserunt Pellarium consulem cum comite Gerardo et Curtevecchia et nobilibus militibus Pisanae civitatis et cum sagittariis et edificatoribus in auxilium imp. F. in obsidione Mediolane: qui maximam expensam et guarnimenta fecerunt. Quos im p. cum toto suo exercitu cum gaudio magno recepit et tenuit. Et 6 Idus Sept. Mediolanenses imp. F. se reddiderunt, et sic omnes nostri cum gratia et bona voluntate imp. et laude et honore Pisas redierunt 6 Kal. Oet.

A. D. 1159 in mense Junio F. imp. venit cum magno exercita in Lombardiam etc. Quapropter Pisani miserunt 15 Kal. Sept. Pellarium consulem cum comite Gerardo et Hermanno filio Paganelli et Curtevecchia, cum quindecim nobilibus militibus melioribus civitatis et cumedificatoribus et cum centum sag ittarii s et duobus sonatoribus foliaree, quos imp.cum ingen ti laetitia et gadiorecepit et tenuit. Unde Mediolanenses hoc audientes tristitiam et timorem magnum habuerunt, et sic postea 8 Idus Sept. imp. se reddiderunt cum fidelitate magna cum trecentis obsidibus et decem milia marchis argenti. Et $6 \mathrm{Idus} \mathrm{S} \mathrm{ept}$. imp. juxta Mediolanum etc. se conoravit.

Dass diese Wiederholung nicht durch ein Schreiberversehen entstanden sein kann, liegt wohl auf der Hand. Die zweite Fassung, so ähnlich sie der ersten ist, hat ein selbständige Einleitung und characteristische Details, die der ersten fehlen. Die Wiederholung muss vom Verfasser herrühren. Wird man es aber einem Zeitgenossen zutrauen, dass er dasselbe Ereigniss mit fast denselben Worten zweimal hintereinander erzäblt? Doch viel eher einem Compilator, der zum 2weiten Male ein etwas abweichendes Excerpt seiner Vorlage giebt ${ }^{1}$ ).

1) So würde sich auch am einfachsten der scheinbare Widerspruch in den Daten der Uebergabe Mailands erklăren. In der Vorlage war zwischen der 
Gleich daneben findet sich eine ähnliche Wiederholung. Es wird zweimal hintereinander erzählt (p. 245), dass der Herzog Welf mit vielen Ehren in Pisa aufgenommen worden sei. Für die Existenz einer Vorlage spricht es auch, wenn p. 247 zum Jahre 1163 der "predictus" consul Petrus aufgeführt wird, ohne dass der Name vorher genannt ist ${ }^{1}$ ). Und schliesslich ein argumentum ex silentio: sollte ein zeitgenössischer, reichstreuer Pisaner unterlassen haben zu erzählen, dass der Kaiser im Jahre 1167 in Pisa gewesen ist?

wirklichen und der feierlichen mit der Krönung des Kaisers verbundenen Uebergabe geschieden. Das erste Mal berücksichtigte der Verfasser nur die feierliche Uebergabe, das zweite Mal gab er das Datum der wirklichen Uebergabe und hob von dem feierlichen Act nur die Krönung herror.

1) Hier kann allerdings auch eine Lücke in Text angenommen werden. 